Review Article

\title{
An Overview of Long Noncoding RNAs Involved in Bone Regeneration from Mesenchymal Stem Cells
}

\author{
Shuping Peng $\mathbb{D}^{1,2}$ Lihua Cao, ${ }^{1,2}$ Shiwei He $\mathbb{D}^{1,2}$ Yancheng Zhong, ${ }^{1,2}$ Haotian Ma, ${ }^{1,2}$ \\ Yanru Zhang, ${ }^{1,2}$ and Cijun Shuai $\mathbb{1}^{3,4}$ \\ ${ }^{1}$ Hunan Provincial Tumor Hospital and The Affiliated Tumor Hospital of Xiangya School of Medicine, Basic Medicine College, \\ Central South University, Changsha 410013, China \\ ${ }^{2}$ Cancer Research Institute, School of Basic Medical Science, Central South University, Changsha 410078, China \\ ${ }^{3}$ School of Energy and Mechanical Engineering, Jiangxi University of Science and Technology, Nanchang, Jiangxi, China \\ ${ }^{4}$ State Key Laboratory of High Performance Complex Manufacturing, College of Mechanical and Electrical Engineering, \\ Central South University, Changsha, Hunan, China \\ Correspondence should be addressed to Cijun Shuai; shuai@csu.edu.cn
}

Received 12 August 2017; Revised 16 November 2017; Accepted 3 December 2017; Published 28 January 2018

Academic Editor: Silvia Brunelli

Copyright (c) 2018 Shuping Peng et al. This is an open access article distributed under the Creative Commons Attribution License, which permits unrestricted use, distribution, and reproduction in any medium, provided the original work is properly cited.

\begin{abstract}
Bone regeneration is very important for the recovery of some diseases including osteoporosis and bone fracture trauma. It is a multiple-step- and multiple-gene-involved complex process, including the matrix secretion and calcium mineralization by osteoblasts differentiated from mesenchymal stem cells (MSCs) and the absorption of calcium and phosphorus by osteoclasts differentiated from hematopoietic stem cells. Long noncoding RNAs (lncRNAs) are a family of transcripts longer than $200 \mathrm{nt}$ without or with very low protein-coding potential. Recent studies have demonstrated that lncRNAs are widely involved in the regulation of lineage commitment and differentiation of stem cells through multiple mechanisms. In this review, we will summarize the roles and molecular mechanism of lncRNAs including H19, MALAT1, MODR, HOTAIR, DANCR, MEG3, HoxA-AS3, and MIAT in osteogenesis ossification; lncRNA ZBED3-AS1 and CTA-941F9.9, DANCR, and HIT in chondrogenic differentiation; and lncRNA DANCR in osteoclast differentiation. These findings will facilitate the development and application of novel molecular drugs which regulate the balance of bone formation and absorption.
\end{abstract}

\section{Introduction}

The bone regeneration after bone fracture trauma or other diseases is a process participated by a well-organized system of the synergistic effect of MSCs, immune cells, and osteoclasts. Osteoclasts absorb the organic and inorganic compounds released from the impaired bone, during which the degraded compound matrix goes into the bloodstream in the form of $\mathrm{Ca}^{+},(\mathrm{PO} 4) 3^{-}$, and so on for recycling $[1,2]$. Meanwhile, the cytokines after the damage process initiate the osteogenic differentiation of MSCs. MSCs gradually differentiate into osteoprogenitors, preosteoblasts, and osteoblasts. The well-differentiated osteoblasts synthesize and secrete the matrix and thus induce the initiation of bone formation. MSC-mediated bone regeneration and osteoclastmediated bone resorption are the two core processes of bone regeneration and repair. The process of osteogenic differentiation of MSCs is mainly regulated by tissue-specific transcriptional regulators and epigenetic factors $[3,4]$. On the one hand, in the corporate induction of BMPs, Wnt $/ \beta$ FGF, and other growth factors, related molecules in the signal pathways such as BMPs/Smads [5], Wnt/ $\beta$-catenin [6] and MAPK/p38 [7], and transcription factors RUNX2 [8] and OSX [9] are activated, increasing the expression of osteoblast-specific genes (OPN, OCN, ALP, and COL1A1); eventually, MSCs differentiate into osteoblasts. On the other hand, epigenetic modulation including DNA methylation, histone modification, and noncoding RNA regulation also 
exerts a role in the regulation of osteogenic differentiation of MSCs. The regulation of DNA methylation and histone modification has been well understood. For example, Dansranjavin et al. found that the osteocalcin of undifferentiated stem cells was hypermethylated. However, in mature osteocytes, the degree of methylation was reduced and the expression levels of osteocalcin were increased [10]. Hsiao et al. observed that transfection of human bone marrow MSCs with the methylated thyroid hormone receptor interactor 10 (Trip 10) promoter resulted in cytosine methylation at the promoter region and downregulation of Trip10 expression, in which accelerating MSCs differentiate into neurons and osteoblasts [11]. Histone acetylation and methylation are another important epigenetic mechanisms in the process of osteogenesis [12-15]. Studies have shown that the BMP signaling pathway promotes osteogenic differentiation by regulating the acetylation of $\mathrm{H} 3 \mathrm{~K} 9$. Shen et al. observed that $\mathrm{H} 3 \mathrm{~K} 4$ methylation decreased while $\mathrm{H} 3 \mathrm{~K} 9$ acetylation increased during the osteogenic differentiation of ROS17/ 2.8 osteosarcoma cells and normal osteoblasts by using CHIP-seq techniques [16]. The roles and related mechanisms of miRNAs in bone development and balance have been reviewed [17-19]. However, the regulation of lncRNAs on bone regeneration has not well summarized.

Long noncoding RNAs (lncRNAs) belong to a family of transcripts longer than $200 \mathrm{nt}$ without or with very low protein-coding potential. In the human genome, 15,787 lncRNA transcripts from 14,470 lncRNA genes have been identified, while the GENCODE annotation is constantly being updated $[20,21]$. It is believed that lncRNAs are a transcriptional noise for a long time, which are a byproduct of RNA polymerase II transcription without biological function. However, recent studies have found that lncRNAs play a crucial role in regulating nuclear chromatin structure and gene expression in the developmental process and are also an active participant in disease occurrence and development [22-24]. Except extensive and constitutive expression of partial lncRNAs, most lncRNAs are specifically expressed during the cell tissue developmental stage. In general, the general expression levels of lncRNA are lower than those of mRNA. Some lncRNAs are located in the cell nucleus and some in the cytoplasm. Compared with miRNA, the interspecies homology similarity of lncRNAs is relatively lower, but there is a certain degree of conservation in its promoter region and exon area, which indicates the function of lncRNAs is relatively conservative. The transcripts produced from the $4 \sim 9 \%$ sequence of the mammalian genome sequence are lncRNAs (corresponding protein-coding portion is $1 \%$ ). Despite that the recent advances on lncRNA have progressed rapidly, the functions of most of lncRNAs are still unclear.

\section{Classification and Characteristics of lncRNAs}

According to the genomic location, lncRNAs can be classified into five types: sense, antisense, bidirectional, intronic, and intergenic [3, 4, 25]. Many lncRNAs have conserved secondary structures, alternative splicing, and subcellular localization. The conservativeness and specificity indicate that they are functional [26]. IncRNAs possess the following characteristics: (1) The length of transcripts is 200$100,000 \mathrm{nt}$, with a similar structure to that of the mRNA. After splicing, there is a structure with a poly $(\mathrm{A})$ tail and a promoter. During differentiated processes, there are a dynamic expression mechanism and alternative splicing that form different lncRNAs [27]. (2) Generally, lncRNAs have noncoding potentials, but some lncRNAs can encode some short peptides [28]. (3) They have low conservation [29]. (4) They are tissue-specific and spatiotemporal-specific. The amount of lncRNAs expressed in different tissues was different, and the expression of lncRNAs was different in the same tissues but different status [30]. (5) The abundance of different lncRNAs is various in different cells [31].

\section{Modes of Action of lncRNAs}

With the gradual knowledge of lncRNA functions, the mechanism of IncRNA interaction with targets has become a hot topic. Early identification of in situ regulation is the only mechanism in which lncRNAs silence the transcription of adjacent genes by recruiting chromatin-modifying complexes. The mechanism of lncRNAs is very complex and has not yet been fully understood. According to the current research, the mechanism of lncRNAs could be summarized as the four levels (epigenetic, transcriptional, and posttranscriptional regulation and other specific regulation modes).

3.1. IncRNAs Mediate Epigenetic Modifications. IncRNAs can recruit a chromatin remodeling complex to specific sites and then regulate the expression of targeting genes. For example, HOTAIR derived from the HOXC loci recruits the chromatin remodeling complex PRC2 and locates it to the HOXD site, thereby inducing the parent genetic silencing of the HOXD loci [32-34]. Similarly, lncRNAs Xist [35] and Kcnq1ot $[36,37]$ can be recruited by the remodeling complexes such as methyltransferase Ezh2 or G9a to realize epigenetic silence of related genes.

3.2. IncRNAs Regulate Transcriptional Expression. IncRNAs can silence gene expression at the transcriptional level through a variety of mechanisms. IncRNAs can interfere with the transcription of adjacent genes. For example, in yeast, the transcription of the SER3 gene is affected by its upstream lncRNA SRG1 [38]. IncRNAs can interfere with gene expression by blocking the promoter region. For instance, lncRNA DHFR can form an RNA-DNA3 helix structure in the promoter region of the DHFR gene [39], inhibiting the binding of the transcription factor TFIID and thereby inhibiting DHFR gene expression. Moreover, lncRNA can interact with RNA-binding proteins and target to the promoter region, regulating gene expression. For instance, lncRNA located in the upstream of the CCND1 promoter can regulate the activity of the RNA-binding protein TLS and affect the expression of CCND1 [40]. Besides, lncRNAs regulate the activity of transcription factors. IncRNA Evf2 can form transcriptional complexes with the transcription factor Dlx2 to activate Dlx6 expression $[41,42]$. At last, lncRNAs can control gene expression by regulating the basic transcription factor. For 
instance, Alu RNA can realize extensive gene suppression by inhibiting RNA polymerase II [43].

3.3. IncRNAs Mediate Posttranscriptional Regulation. lncRNA can form double-stranded RNA complexes with mRNA at the posttranscriptional level to mask the major cis-acting elements of mRNA, thereby regulating gene expression. For example, lncRNA Zeb2 (Sip1) is able to form a double strand at the $5^{\prime}$ end shear site of an intron of the mRNA transcribed by the HOX site, thereby preventing the intron from being sheared. The region contains ribosomebinding sites which are necessary for the expression of Zeb2 protein, and Zeb2 antisense RNA can increase the expression of Zeb2 protein in this way. This example shows that lncRNAs can guide alternative splicing of mRNA isoforms. lncRNAs compete with mRNA to bind miRNA-binding sites, leading to the upregulation of miRNA target molecules. LncMD as a spongy molecule isolates miR-125b binding to the target molecule IGF mRNA, promoting MSC differentiation into muscle cells [44].

3.4. Other Specific Regulation Modes. In addition, the renaturation (annealing) of lncRNAs has a targeting effect, allowing protein receptor complexes to recognize the mRNA transcripts of the sense chain. This mode resembles the RNA-induced silencing complex (RISC) targeting mRNA through siRNA. Double-stranded RNA derived from complementary transcripts and even lncRNA, combined with extended internal hairpin structure, can be processed into endogenous siRNA to silence gene expression.

\section{IncRNAs in Bone Development and Homeostasis}

The formation of new bone is induced from MSCs via lineage commitment, which successively form osteoprogenitor cells, preosteoblasts, mature osteoblasts, and osteocytes. These major regulatory mechanisms, including tissue-specific transcription factors and regulatory molecules, mediated bone matrix synthesis, bone remodeling, and mineralizationrelated and repair-related gene expression. These osteogenic activities are simultaneously regulated by genetic and epigenetic levels. Epigenetic regulation includes DNA methylation, histone modification, and miRNA and lncRNA regulation. miRNA regulation for bone function and repair process has been summarized before.

Numerous experiments have shown that $\operatorname{lncRNAs}$ play a role in these processes. Classification and functional analyses also show that lncRNAs are involved in the lineage differentiation of MSCs into muscle cells, adipocytes, chondrocytes, and osteoblasts. Many scientists have focused on how lncRNAs play a role in stem cell differentiation for the past few years. Here, we will concentrate on the expression of lncRNAs in osteogenic differentiation of MSCs (Table 1). In addition, we will also analyze the role of lncRNAs in bone and cartilage differentiation, as well as the role and balance of bone, cartilage, and osteoclasts.

\section{Global Transcriptomic Analyses Identify lncRNA Profiles}

Zuo et al. [45] firstly published the earliest report about osteogenesis-related lncRNAs identified at 2013. They found that the expression profile of lncRNAs from C3H10T1/2 MSCs was changed under BMP2 induction. At the same time, they identified 116 differentially expressed lncRNAs and these lncRNAs positively regulate the expression of its adjacent genes, which indicated that lncRNAs regulated osteogenesis under the synergistic effect of adjacent genes. Song et al. utilized high-throughput RNA sequencing (RNA-seq) data to detect the expression profile of IncRNAs from immortalized MSCs which was induced by osteogenic induction medium for 28 days and thus screened 2597 mRNAs and 574 lncRNAs, of which 351 were known lncRNAs and 217 were novel lncRNAs. 32 novel lncRNAs are the precursor molecules of $m i R-689, m i R-640, m i R-601$, and $m i R-544$. They also constructed 14,275 coexpression relationships in the osteogenesis process, as well as 217 gene regulatory networks between the novel IncRNA and the mRNA [46] .

$\mathrm{Qu}$ et al. utilized high-throughput expression profiles (30,586 lncRNAs and 26,109 coding transcripts) to screen the differentially expressed genes of human periodontal ligament stem cells (hPDLSCs), which were, respectively, cultured in growth medium and osteogenic induction medium for 14 days, and screened out 3557 differentially expressed mRNAs, among which 1578 mRNAs were upregulated, 1979 mRNAs downregulated, 994 lncRNAs upregulated, and $1177 \operatorname{lncRNAs}$ downregulated. These lncRNAs (AC078851.1, RP11-45A16.4, XLOC_002932, RP4-613B23.1, and RP11-305L7.6) and mRNAs (ALP, COL1A1, and COL1A20) are upregulated and BMP5 and IL6 are downregulated as verified by Q-PCR indicating that there are 131 pairs of lncRNA-mRNA regulatory relationships and 262 pairs of positive regulatory relationships, and MAPK, VEGF, and TGF- $\beta$ signaling pathways were mainly involved in the regulation during osteogenic differentiation process [47].

Zhang et al. reported the human BMSCs derived from 18- to 20-year-old healthy male bone marrow cultured in osteogenic induction medium for 7 days and screened them with high-throughput human transcription microarray (Affymetrix, covering more than 285,000 coding and 40,000 noncoding transcripts). They screened out 1269 differentially expressed mRNAs (among which 648 were upregulated and 621 were downregulated) and 1408 lncRNAs, and MAPK, JAK-STAT, Toll-like receptor, and TGF- $\beta$ signal pathways were found to participate in osteogenic differentiation of hBMSCs. GPX3, TLR2, BDKRB1, FBXO5, BRCA1, MAP3K8, SCARB1, and $6 \operatorname{lncRNAs}$ (XR_111050, NR_024031, FR374455, FR401275, FR406817, and FR148647) played a key role in osteogenic process, and lncRNA XR_111050 promoted osteogenic differentiation of mesenchymal stromal cells [48]. It can be confirmed that lncRNAs play an important role in osteogenesis differentiation, and the current studies have identified a number of differentially expressed lncRNAs. However, the mechanisms of most lncRNAs regulating the osteogenesis process remain to be understood and explored. 


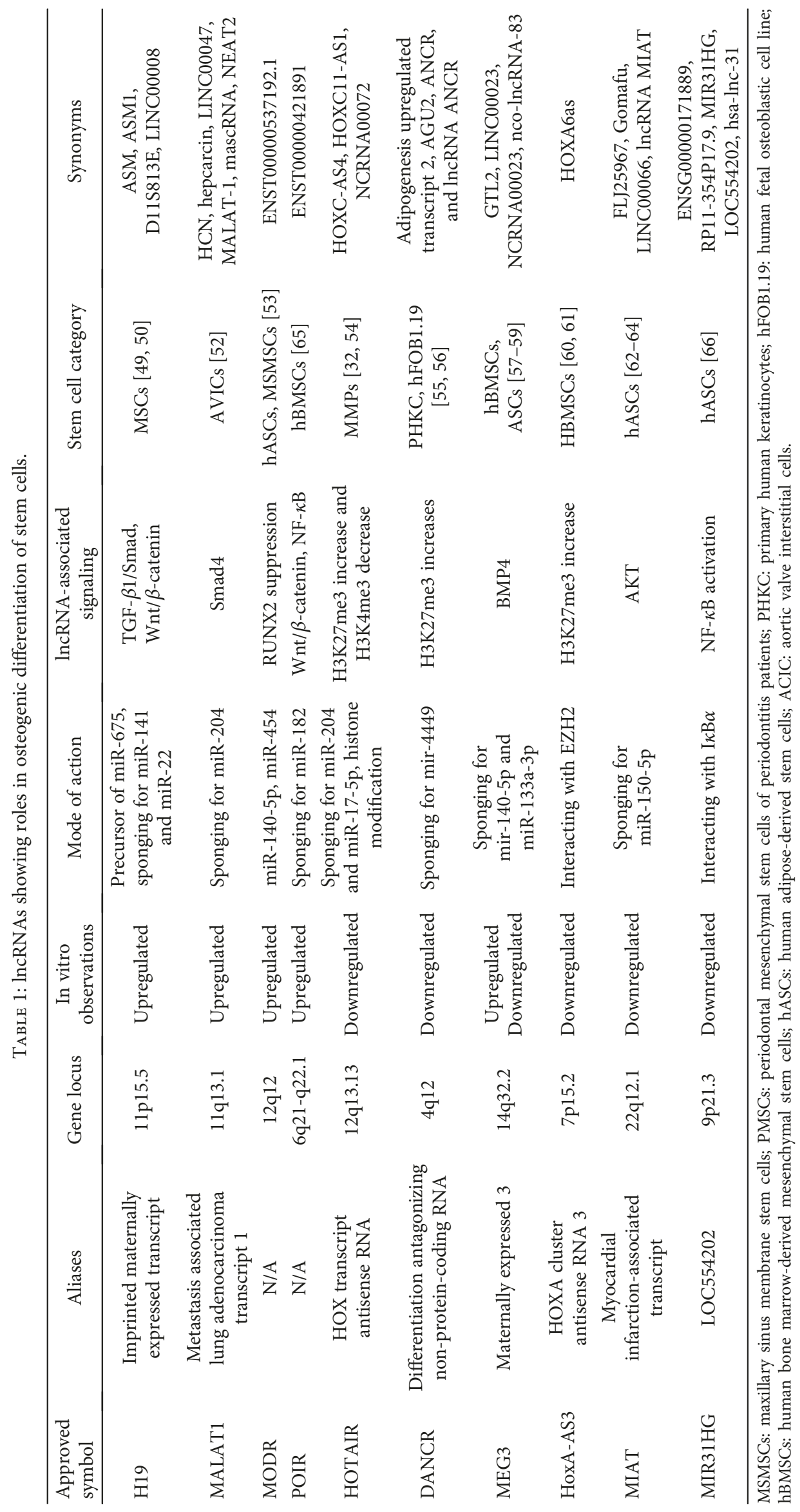




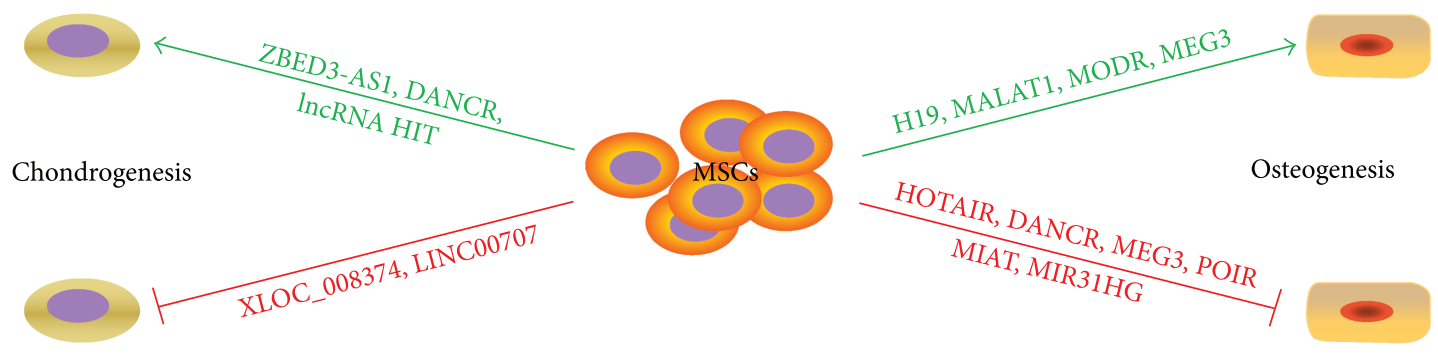

FIGURE 1: lncRNAs that promote osteogenic differentiation of stem cells.

\section{IncRNAs Are Functionally Involved in Bone Development and Homeostasis}

\subsection{IncRNAs That Promote Osteogenic Differentiation of Stem Cells}

6.1.1. H19. H19 (imprinted maternally expressed transcript) is one of the highly upregulated genes during the induction of primitive stem cells with osteogenic induction medium. It is located on $11 \mathrm{p} 15.5$ and is $2.3 \mathrm{~kb}$ in length and is conserved in evolution and plays an important role in regulating biological functions. $H 19$ is the precursor of $m i R-675$ which can generate two mature miRNAs ( $m i R-675-5 p$ and $m i R-$ $675-3 p)$ by Drosha and Dicer with splicing-dependent modes. H19 and miR-675 were upregulated during osteogenic differentiation of human MSCs. miR-675 not only downregulates TGF- $\beta 1$ but also inhibits Smad3 phosphorylation and downregulates $\mathrm{HDAC} 4 / 5$ leading to reduced HDACs to be recruited to the promoter of osteogenesisspecific runt-related transcription factor 2 (Runx2) [49]. Liang et al. found that $H 19$, acting as an endogenous competitive ceRNA for $m i R-141$ and $m i R-22$, directly binds to $m i R$ 141 and $m i R-22$ preventing the inhibition of $m i R-141$ and miR-22 on Wnt/beta-catenin, thereby promoting osteogenic differentiation [50]. Huang et al. reported that $H 19$ and miR-675 overexpression inhibited adipogenic differentiation. miR-675 targets to the $3^{\prime}$-UTR region of histone deacetylase HDAC4 6 that downregulates the expression of HDAC4 6 which is the essential molecules of fat differentiation. Recent studies have shown that H19 plays a critical role in embryonic placenta growth and cell differentiation [51] (Figure 1).

6.1.2. MALAT1. MALAT1 (metastasis-associated lung adenocarcinoma transcript 1) is firstly found to be positively correlated with metastasis in lung adenocarcinoma, which is located on 11q13.1 and is 8545 nt in length. Xiao et al. demonstrated that MALAT1 promotes osteogenic differentiation of aortic valve interstitial cells in calcific aortic valve disease (CAVD). Further studies showed that MALAT1 functioned as a sponge molecule of $m i R-204$ and upregulated the expression of Smad4. Smad4 activation promotes the expression of alkaline phosphatase and downstream molecule osteocalcin and thus promotes bone formation and mineralization [52] (Figure 1).

6.1.3. $M O D R$. IncRNA $M O D R$ is an upregulated lncRNA in the process of osteogenesis of maxillary sinus membrane stem cells. Silencing lncRNA MODR can reduce the expression of RUNX2. IncRNA MODR acts as a molecular sponge for binding to $m i R-454$ to relieve its inhibition for $R U N X 2$, thus upregulating $R U N X 2$ expression and promoting osteogenesis [53] (Figure 1).

\subsection{IncRNAs That Inhibit Osteogenic Differentiation}

6.2.1. HOTAIR. HOTAIR (HOX transcript antisense RNA), which is $2.2 \mathrm{~kb}$ in length and is located on $12 \mathrm{q} 13.13$, is a long noncoding RNA formed by HOXC gene transcription. Gene knockout of HOTAIR can lead to homologous transformation and skeletal malformations. HOTAIR can inhibit HOXD, HOTAIR, and imprinted loci, such as DLK1-MEG3 and Igf2-H19. At the same time, it combines with polycomb repressive complex 2 (PRC2, methylated H3k27) and Lsd1 complex (LSD1, demethylated H3K4), resulting in an increase in $\mathrm{H} 3 \mathrm{~K} 27 \mathrm{me} 3$ expression and a decrease in $\mathrm{H} 3 \mathrm{~K} 4 \mathrm{me} 3$ expression, indicating that HOTAIR can enhance the inhibition of HOX and other target genes by chromatin remodeling. Homozygous deletion of the HOTAIR gene in mice leads to lumbosacral bone turnover and metacarpal and carpal bone fusion, which is similar to the ectopic overexpression of $H O X D$ in transgenic mice with an increase in HOXD10 and HOXD11 expression, suggesting that HOTAIR transregulated HOXD gene expression by recruiting polycomb and inhibiting the PRC2 complex targeting HOXD sites [32]. Compared with osteoarthritis, the expression of the long noncoding RNA HOTAIR from MSCs of patients with nontraumatic necrosis of the femoral head is upregulated while that of $m i R-17-5 p$ was downregulated. Knockdown of HOTAIR by siRNA leads to an increase in $m i R-17-5 p$ expression and a decrease in its target gene smad7, while RUNX2, COLA1 mRNA, and alkaline phosphatase activities were upregulated. Thus, HOTAIR reduces the expression of $m i R-17-5 p$ by reducing Smad7 and then inhibiting osteogenic differentiation [54] (Figure 2).

6.2.2. DANCR. DANCR (differentiation antagonizing nonprotein-coding RNA) was initially found to be downregulated during calcium ion-induced primary human keratinocyte differentiation by RNA-seq. Human DANCR is located on $4 \mathrm{q} 12$ and is $915 \mathrm{bp}$ in length, having 3 exons and an miR-4449-binding site, and located at the downstream of $54.8 \mathrm{~kb}$ and $28.7 \mathrm{~kb}$ are the USP and ERVMER34-1 genes. Zhu and $\mathrm{Xu}$ later found that downregulation of DANCR promoted osteogenic differentiation of the human fetal osteoblastic cell line hFOB1.19 cultured in osteogenic induction 


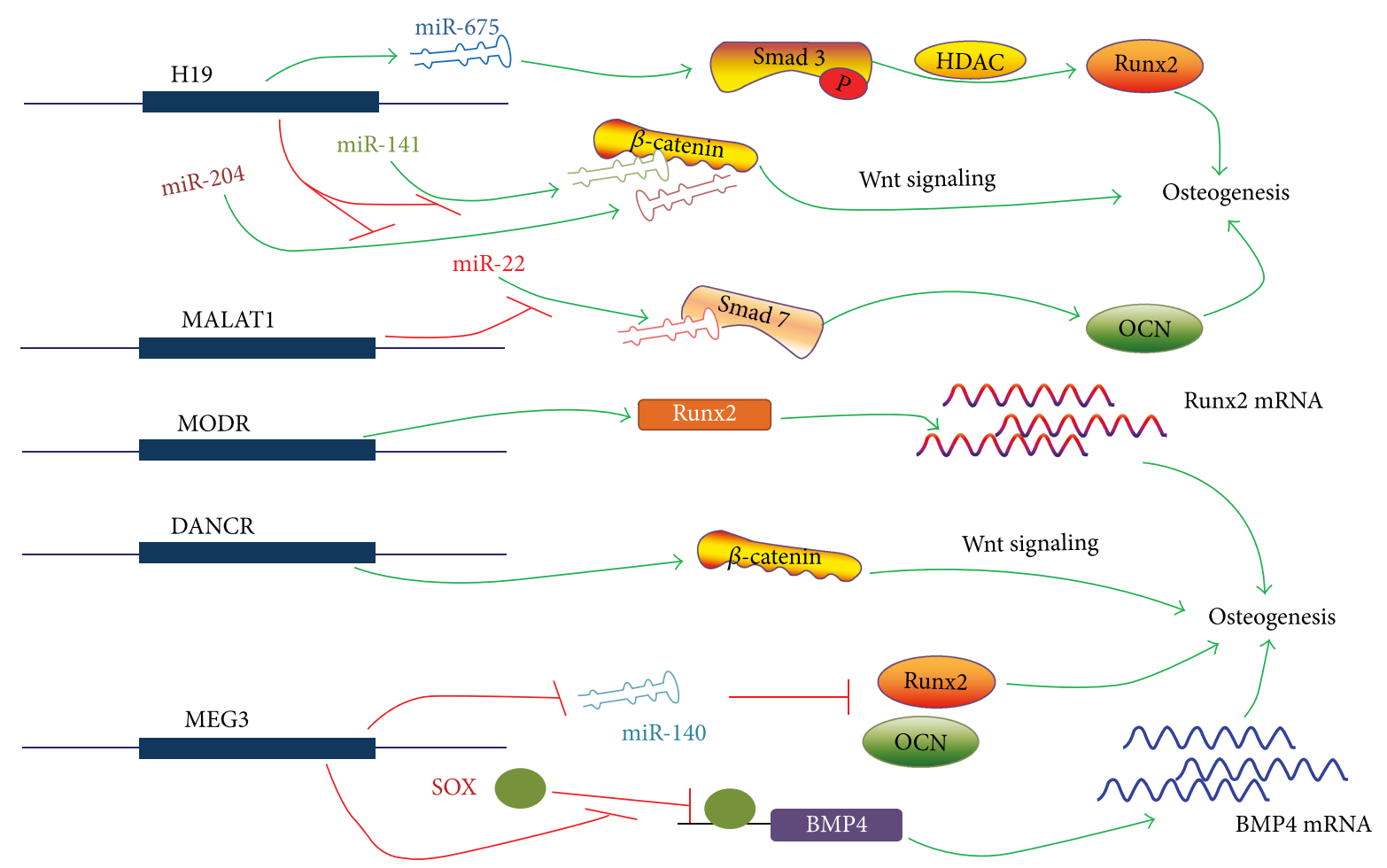

FIGURE 2: lncRNAs that inhibit osteogenic differentiation.

medium. They further found that a transcript with a length of $305 \mathrm{nt}$ at the DANCR $3^{\prime}$ end interacted with EZH2 (enhancer of zeste homolog 2), whereas DANCR recruited $E Z H 2$ to promote $\mathrm{H} 3 \mathrm{~K} 27 \mathrm{me} 3$, which inhibited target gene RUNX2 transcription and osteogenic differentiation [55]. Jia et al. studied that downregulation of DANCR in periodontal ligament stem cells promotes osteogenic differentiation by activating classical Wnt signaling pathways [56] (Figure 2).

6.2.3. MEG3. Zhuang et al. found that MEG3 (maternally expressed 3) promotes the differentiation of bone marrow stem cells (hBMSCs) into osteoblasts in patients with multiple myeloma. MEG3, which is located on $14 \mathrm{q} 32.2$ and is $1595 \mathrm{bp}$ in length, promotes the translation of BMP4 located at the downstream apart few Mbs by preventing the inhibiting effect of SOX2 in the BMP4 promoter region [57]. However, Li et al. detected that MEG3 expression is downregulated during adipose-derived MSC differentiation into adipogenic cells and upregulated during osteogenic differentiation. Knockdown of MEG3 promotes the osteogenic and adipogenic differentiation of human adipose-derived MSCs [58]. The mechanism may be associated with miR140-5p. It also has been reported that MEG3 upregulated $m i R-133 a-3 p$ and inhibited osteogenic differentiation in bone marrow MSCs from patients with postmenopausal osteoporosis [59] (Figure 1).

6.2.4. HoxA-AS3. HoxA-AS3 (HOXA cluster antisense RNA 3) was originally identified as an upregulated molecule in glioma patients, which is located on 7p15.2 and is $3992 \mathrm{nt}$ in length. Later, HoxA-AS3 was found to inhibit osteogenic differentiation of human bone marrow-derived stem cells (hBMSCs) and promote adipogenic differentiation [60]. HoxA-AS3 inhibits RUNX2 transcription by binding to EZH2 and promoting $\mathrm{H} 3 \mathrm{k} 27$ methylation. HoxA-AS3 acts as an epigenetic modified switch to inhibit osteogenic differentiation of MSCs [61] (Figure 2).

6.2.5. MIAT. MIAT (myocardial infarction-associated transcript) is located on chromosome 22q12.1 and downregulated in human adipose-derived stem cells (hASCs) during osteogenic differentiation. Knockdown of MIAT promoted osteogenic differentiation of hASCs in vitro and in vivo. Tumor necrosis factor treatment increases MIAT expression. Knockdown of MIAT can reverse the inhibition of osteogenic differentiation induction by an inflammatory factor. It acts as a sponge molecule of $m i R-150-5 p$ to regulate its binding to the target gene and also acts as an endogenous competitive RNA to form AKT-miR-150-5p feedback loop to regulate oxidative stress and inflammatory factors and to stimulate the functional regulation of human lens epithelial cells [62-64] (Figure 2).

6.2.6. POIR. IncRNA POIR, which is located on chromosome 6 and is $786 \mathrm{nt}$ in length, was found to be expressed differentially in periodontal MSCs from patients with periodontitis and healthy human. Its expression is upregulated during osteogenic differentiation of periodontal membrane stem cells (PMSCs). Further studies have shown that lncRNA $P O I R$ as an endogenous competitive RNA competes for the binding sites of $m i R-182$, leading to an increase in its target 
TABLE 2: lncRNAs showing roles in chondrocyte differentiation of stem cells.

\begin{tabular}{lcccccc}
\hline $\begin{array}{l}\text { Approved } \\
\text { symbol }\end{array}$ & Aliases & $\begin{array}{c}\text { Gene } \\
\text { locus }\end{array}$ & $\begin{array}{c}\text { In vitro } \\
\text { observations }\end{array}$ & Mode of action & $\begin{array}{c}\text { lncRNA-associated } \\
\text { signaling }\end{array}$ & Stem cell category \\
\hline DANCR & $\begin{array}{c}\text { Differentiation } \\
\text { antagonizing } \\
\text { nonprotein }\end{array}$ & $4 \mathrm{q} 12$ & Upregulated & $\begin{array}{c}\text { Sponging for } \\
\text { miR-1305-Smad4 }\end{array}$ & Sox4 binds to the promoter & $\begin{array}{c}\text { SMSCs, hSDSC } \\
{[70,71]}\end{array}$ \\
ZBED3-AS1 & $5 \mathrm{q} 13.3$ & Upregulated & $\begin{array}{c}\text { Directly increases } \\
\text { zbed3 expression }\end{array}$ & $\begin{array}{c}\text { Activates Wnt/ } \beta \text {-catenin } \\
\text { signaling } \\
\text { Increases H3K27ac } \\
\text { or p100 activity }\end{array}$ & $\begin{array}{c}\text { Early limb development } \\
\text { in mice [72, 73] }\end{array}$ \\
\hline
\end{tabular}

gene FOXO1. FOXO1 promotes bone formation by inhibiting the classical Wnt signaling pathway by competing with TCF4 for beta-catenin. And abnormal activation of the NF- $\kappa \mathrm{B}$ pathway during inflammation can increase the level of $m i R$ 182 and decrease the level of lncRNA POIR, which breaks the balance of lncRNA-POIR-miR-182 regulatory network [65] (Figure 1).

6.2.7. MIR31HG. MIR31HG (MIR31 host gene) is located on $9 \mathrm{p} 21$ and is $745 \mathrm{nt}$ in length, which is found downregulated by Jin et al. in the process of induced human adipose-derived stem cell (hASC) osteogenic differentiation. Knockdown of MIR31HG not only promotes the formation of bone but also overcomes the inflammatory inhibition of the osteogenesis process. From the mechanism, it was found that MIR31HG interacted with NF- $\kappa \mathrm{B}$ where p65 subunit bound to the MIR31HG promoter region and promoted MIR31HG expression. MIR31HG binds directly to $\mathrm{I} \kappa \mathrm{B} \alpha$ and participates in NF- $\kappa \mathrm{B}$ activation. Thus, targeting to the MIR31HG-NF- $\kappa \mathrm{B}$ regulatory loop can improve the osteogenic ability of hASCs in inflammatory environments [66] (Figure 2).

\section{IncRNAs Involved in Chondrogenic Differentiation}

Cartilage plays a role in modeling, protecting and supplementing bone tissue during individual development. Chondrocytes are derived from bone pluripotent precursor cells and can form the primordial cartilage and are the main skeleton of the embryo by directional specific regulatory systems and continuous pedigree differentiation processes. This primordial cartilage is then cartilaginous in the cartilage growing plate, involved in bone elongation and temporary drive, or becomes permanent tissue, which is articular cartilage. The fate determinants and differentiation activities of chondrocytes are controlled by many extrinsic and intrinsic clues, which are achieved at the gene expression levels by transcription factors. Tissue repair capacity after injury is limited, often causing rheumatoid arthritis. MSC technology is a promising treatment strategy. SOX9 and RUNX2/3, as well as TWIST1, SOX5/6, and MEF2C/D, are the main transcription factors that regulate cartilage differentiation [67].

7.1. ZBED3-AS1. Ou et al. studied that the IncRNA expression profile changed during induced cartilage differentiation process in hBMSCs, which suggested that $2166 \operatorname{lncRNAs}$ were upregulated and 1472 lncRNAs were downregulated; the expression of ZBED3-AS1 (ZBED3 antisense RNA 1) and CTA-941F9.9 was verified by Q-PCR. These results indicate that lncRNAs are involved in MSC cartilage differentiation [68] (Table 2).

7.2. DANCR. Zhang et al. found that SOX4 in synoviumderived MSCs (SMSCs) can promote SMSC proliferation and differentiate into chondrocytes by upregulating $\operatorname{lncRNA}$ DANCR. DANCR (differentiation antagonizing non-proteincoding RNA) directly combines with myc, Smad3, and STAT3 mRNA and regulates their stability. SMSC proliferation depends on myc. DANCR activates SMSC differentiation into chondrocytes by increasing Smad3 [69]. Zhang et al. found DANCR promoted chondrogenesis by downregulating the expression of miR-1305 which resulted in the decreased expression of Smad 4 and activation of the TGF- $\beta$ pathway in human synovium-derived stem cells (SMSCs) $[70,71]$ (Table 2).

7.3. HIT. Carlson et al. and Wang et al. found that lncRNA HIT was highly expressed in E11 mouse embryos, which is located in the nucleus and formed complexes with p100 and CBP. CHIRP-seq analysis revealed that the lncRNA-HIT-p100/CBP complex was associated with multiple sites in the mouse genome and exerted its role in cartilage differentiation. Silencing HIT with specific siRNA leads to decreased p100 activity and decreased H3K27ac, so lncRNA HIT plays an integral role in cartilage differentiation $[72,73]$ (Table 2).

\section{IncRNAs Involved in Osteoclastogenesis}

Bone formation is a dynamic and continuous experience shaping, repair, and reconstruction process. Bone balance is mainly maintained which is dependent on osteoblasts involved in the formation of new bone and on osteoclasts involved in bone resorption. Osteoclasts are multinucleated cells derived from hematopoietic stem cells or monocyte/ macrophage precursor cells. Osteoclast differentiation includes multiple stages. Dou et al. identified the transcriptional changes of RAW264.7 cells during osteoclast differentiation induced by RANKL $(100 \mathrm{ng} / \mathrm{mL})$ and M-CSF $(50 \mathrm{ng} / \mathrm{mL})$ for $24 \mathrm{~h}, 72 \mathrm{~h}$, and $96 \mathrm{~h}$. A series of changes were identified for circRNA, miRNA, IncRNA, and mRNA. 


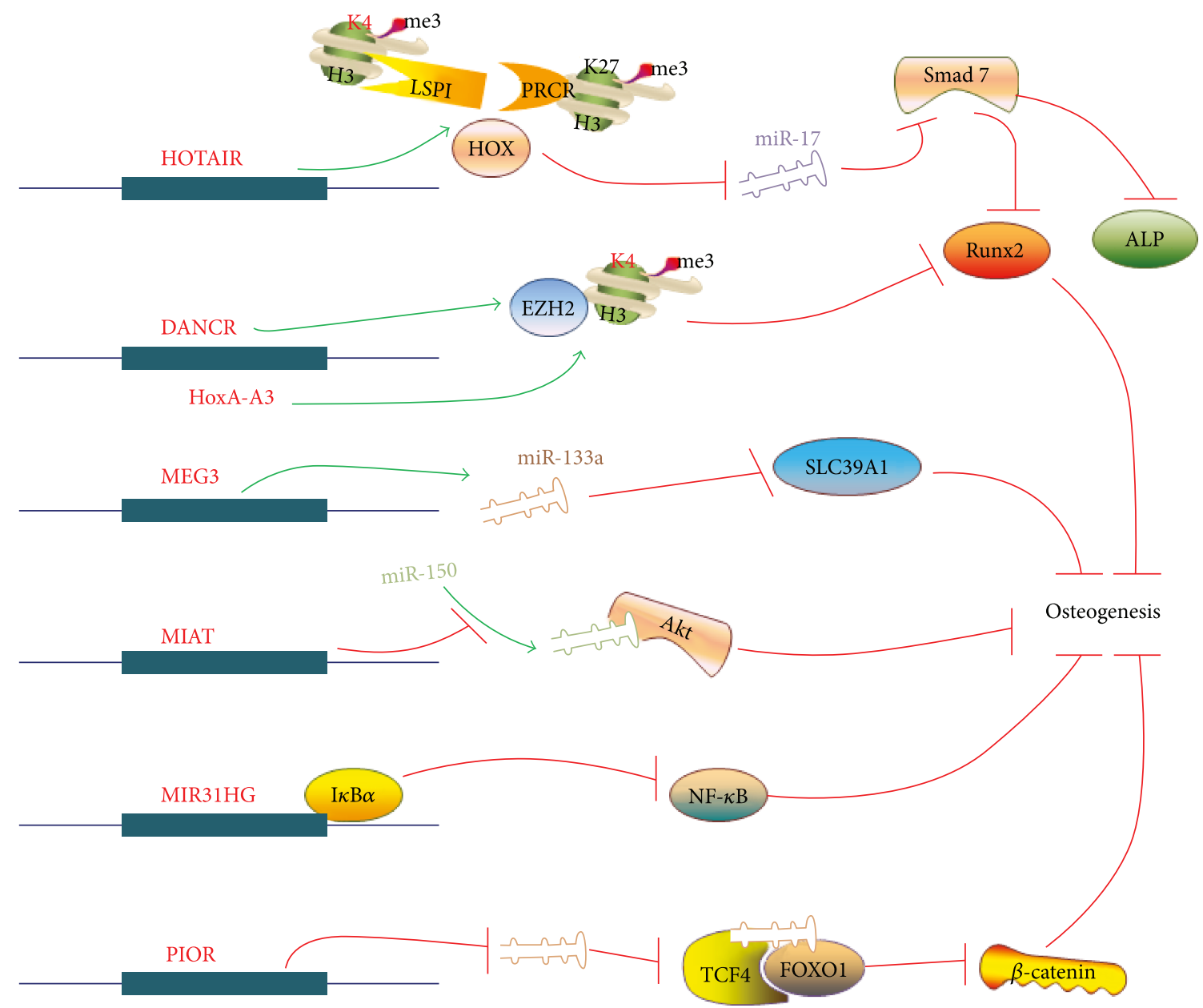

FIgURE 3: lncRNAs that contribute to lineage-specific phenotypes of MSCs.

The research group constructed 142 pairs of correlation between lncRNA and mRNA [74] and found that lncRNAs are also involved in the regulation of hematopoietic stem cell differentiation into osteoclasts. Osteoporosis is a common disease associated with reduced bone mineralization, which is mainly due to osteoblastic bone resorption exceeding bone formation function of osteoblasts. Tong et al. reported that IncRNA DANCR was involved in mononuclear cell formation in peripheral blood and was associated with human osteoporosis. DANCR promotes IL6 and TNF$\alpha$ expression and increases bone resorption. These results suggest that lncRNAs are involved in bone resorption processes of osteoclasts [75] (Figure 3).

\section{Concluding Remarks}

In this manuscript, we summarized the long noncoding RNAs which play an important role in the osteogenic differentiation (H19, MALAT1, MODR, etc.), cartilage differentiation (ZBED3-AS1, DANCR, and HIT) from MSCs, and osteoclast differentiation (DANCR) from hematopoietic stem cells and mononuclear progenitor cells. The mechanism has been demonstrated. Compared with coding protein and small RNA, the knowledge of lncRNAs is only at an initial stage; functions and regulation mechanisms of which remain to be further elucidated. At present, we get to know about the functions and regulatory molecular mechanism through traditional techniques including in situ hybridization technology, overexpression technology, luciferase reporter gene system, and gene silencing technology by siRNA and Crisp/ Cas9. At the same time, the development of some new technologies, such as CLIP (cross-linking immunoprecipitation) [76-78], RIP (RNA-binding protein immunoprecipitation) [79], RNA pulldown [80], CLASH (cross-linking, ligation, and sequencing of hybrids) [81], and ChIRP (chromatin isolation by RNA purifications) [82], has also provided a new platform for studying the networks involving proteins and RNA. With the development of more high-throughput screening technologies, such as microarray chip hybridization, combining the new generation of high-throughput sequencing technology with bioinformatics prediction tools, people will be able to find those with important regulatory functions more quickly and efficiently. The understanding, development, and application of the novel lncRNAs in the field of regeneration and repair will also present a new blueprint for a better and healthier life. 


\section{Conflicts of Interest}

The authors declare no conflict of interest. This paper is not submitted or under consideration for publication elsewhere.

\section{Authors' Contributions}

This manuscript has been read and approved by all authors.

\section{Acknowledgments}

The authors would like to thank all laboratory members for their critical discussion of this manuscript and apologize to those not mentioned due to space limitations. This work was supported by the Natural Science Foundation of China (81572577), the Program of Introducing Talents of Discipline to Universities (111-2-12), the Natural Science Foundation of Hunan Province (2016JJ1027), the Project of Innovation-Driven Plan of Central South University (2016CX023), the Open-End Fund for the Valuable and Precision Instruments of Central South University, and the Fundamental Research Funds for the Central Universities of Central South University (2017zzts371).

\section{References}

[1] K. Matsuo, "Cross-talk among bone cells," Current Opinion in Nephrology and Hypertension, vol. 18, no. 4, pp. 292-297, 2009.

[2] W. J. Boyle, W. S. Simonet, and D. L. Lacey, "Osteoclast differentiation and activation," Nature, vol. 423, no. 6937, pp. 337342, 2003.

[3] T. Kunej, J. Obsteter, Z. Pogacar, S. Horvat, and G. A. Calin, "The decalog of long non-coding RNA involvement in cancer diagnosis and monitoring," Critical Reviews in Clinical Laboratory Sciences, vol. 51, no. 6, pp. 344-357, 2014.

[4] L. Zhu, J. Zhu, Y. Liu et al., "Methamphetamine induces alterations in the long non-coding RNAs expression profile in the nucleus accumbens of the mouse," BMC Neuroscience, vol. 16, no. 1, p. 18, 2015.

[5] J. Huang, S. X. Yuan, D. X. Wang et al., "The role of COX-2 in mediating the effect of PTEN on BMP9 induced osteogenic differentiation in mouse embryonic fibroblasts," Biomaterials, vol. 35, no. 36, pp. 9649-9659, 2014.

[6] F. Yang, D. Yang, J. Tu, Q. Zheng, L. Cai, and L. Wang, "Strontium enhances osteogenic differentiation of mesenchymal stem cells and in vivo bone formation by activating Wnt/ catenin signaling," Stem Cells, vol. 29, no. 6, pp. 981-991, 2011.

[7] X. Zhang, C. Zhou, X. Zha et al., "Apigenin promotes osteogenic differentiation of human mesenchymal stem cells through JNK and p38 MAPK pathways," Molecular and Cellular Biochemistry, vol. 407, no. 1-2, pp. 41-50, 2015.

[8] C. Zhang, "Transcriptional regulation of bone formation by the osteoblast-specific transcription factor Osx," Journal of Orthopaedic Surgery and Research, vol. 5, no. 1, p. 37, 2010.

[9] S. Vimalraj, B. Arumugam, P. J. Miranda, and N. Selvamurugan, "Runx2: structure, function, and phosphorylation in osteoblast differentiation," International Journal of Biological Macromolecules, vol. 78, pp. 202-208, 2015.

[10] T. Dansranjavin, S. Krehl, T. Mueller, L. P. Mueller, H. J. Schmoll, and R. H. Dammann, "The role of promoter CpG methylation in the epigenetic control of stem cell related genes during differentiation," Cell Cycle, vol. 8, no. 6, pp. 916-924, 2009.

[11] S. H. Hsiao, K. D. Lee, C. C. Hsu et al., "DNA methylation of the Trip10 promoter accelerates mesenchymal stem cell lineage determination," Biochemical and Biophysical Research Communications, vol. 400, no. 3, pp. 305-312, 2010.

[12] H. Wu, J. A. Gordon, T. W. Whitfield et al., "Chromatin dynamics regulate mesenchymal stem cell lineage specification and differentiation to osteogenesis," Biochimica et Biophysica Acta (BBA) - Gene Regulatory Mechanisms, vol. 1860, no. 4, pp. 438-449, 2017.

[13] M. B. Meyer, N. A. Benkusky, B. Sen, J. Rubin, and J. W. Pike, "Epigenetic plasticity drives adipogenic and osteogenic differentiation of marrow-derived mesenchymal stem cells," Journal of Biological Chemistry, vol. 291, no. 34, pp. 17829-17847, 2016.

[14] P. Zhang, Y. Liu, C. Jin et al., "Histone H3K9 acetyltransferase PCAF is essential for osteogenic differentiation through bone morphogenetic protein signaling and may be involved in osteoporosis," Stem Cells, vol. 34, no. 9, pp. 2332-2341, 2016.

[15] Y. Liu, X. L. Zhang, L. Chen et al., "Epigenetic mechanisms of bone regeneration and homeostasis," Progress in Biophysics and Molecular Biology, vol. 122, no. 2, pp. 85-92, 2016.

[16] J. Shen, H. Hovhannisyan, J. B. Lian et al., "Transcriptional induction of the osteocalcin gene during osteoblast differentiation involves acetylation of histones $\mathrm{H} 3$ and $\mathrm{H} 4$," Molecular Endocrinology, vol. 17, no. 4, pp. 743-756, 2003.

[17] C. Huang, J. Geng, and S. Jiang, "MicroRNAs in regulation of osteogenic differentiation of mesenchymal stem cells," Cell and Tissue Research, vol. 368, no. 2, pp. 229-238, 2017.

[18] J. B. Lian, G. S. Stein, A. J. van Wijnen et al., "MicroRNA control of bone formation and homeostasis," Nature Reviews Endocrinology, vol. 8, no. 4, pp. 212-227, 2012.

[19] S. Fang, Y. Deng, P. Gu, and X. Fan, "MicroRNAs regulate bone development and regeneration," International Journal of Molecular Sciences, vol. 16, no. 4, pp. 8227-8253, 2015.

[20] M. J. Hangauer, I. W. Vaughn, and M. T. McManus, "Pervasive transcription of the human genome produces thousands of previously unidentified long intergenic noncoding RNAs," PLoS Genetics, vol. 9, no. 6, article e1003569, 2013.

[21] B. H. You, S. H. Yoon, and J. W. Nam, "High-confidence coding and noncoding transcriptome maps," Genome Research, vol. 27, no. 6, pp. 1050-1062, 2017.

[22] S. Gayen and S. Kalantry, "Chromatin-enriched lncRNAs: a novel class of enhancer RNAs," Nature Structural \& Molecular Biology, vol. 24, no. 7, pp. 556-557, 2017.

[23] X. Q. Wang and J. Dostie, "Reciprocal regulation of chromatin state and architecture by HOTAIRM1 contributes to temporal collinear HOXA gene activation," Nucleic Acids Research, vol. 45, no. 3, pp. 1091-1104, 2017.

[24] D. H. Kim and S. Sung, "Vernalization-triggered intragenic chromatin loop formation by long noncoding RNAs," Developmental Cell, vol. 40, no. 3, pp. 302-312.e4, 2017.

[25] L. Ma, V. B. Bajic, and Z. Zhang, "On the classification of long non-coding RNAs,” RNA Biology, vol. 10, no. 6, pp. 925-933, 2013.

[26] J. J. Quinn and H. Y. Chang, "Unique features of long noncoding RNA biogenesis and function," Nature Reviews Genetics, vol. 17, no. 1, pp. 47-62, 2016. 
[27] D. Zhang, M. Xiong, C. Xu, P. Xiang, and X. Zhong, "Long noncoding RNAs: an overview," Methods in Molecular Biology, vol. 1402, pp. 287-295, 2016.

[28] N. Rion and M. A. Ruegg, "LncRNA-encoded peptides: more than translational noise?," Cell Research, vol. 27, no. 5, pp. 604-605, 2017.

[29] L. Yang, J. E. Froberg, and J. T. Lee, "Long noncoding RNAs: fresh perspectives into the RNA world," Trends in Biochemical Sciences, vol. 39, no. 1, pp. 35-43, 2014.

[30] E. D. Kim and S. Sung, "Long noncoding RNA: unveiling hidden layer of gene regulatory networks," Trends in Plant Science, vol. 17, no. 1, pp. 16-21, 2012.

[31] A. J. Blythe, A. H. Fox, and C. S. Bond, "The ins and outs of lncRNA structure: how, why and what comes next?," Biochimica et Biophysica Acta, vol. 1859, no. 1, pp. 46-58, 2016.

[32] L. Li, B. Liu, O. L. Wapinski et al., "Targeted disruption of Hotair leads to homeotic transformation and gene derepression," Cell Reports, vol. 5, no. 1, pp. 3-12, 2013.

[33] A. R. Amandio, A. Necsulea, E. Joye, B. Mascrez, and D. Duboule, "Hotair is dispensible for mouse development," PLoS Genetics, vol. 12, no. 12, article e1006232, 2016.

[34] J. L. Rinn, M. Kertesz, J. K. Wang et al., "Functional demarcation of active and silent chromatin domains in human HOX loci by noncoding RNAs," Cell, vol. 129, no. 7, pp. 13111323, 2007.

[35] A. Monfort, G. Di Minin, A. Postlmayr et al., "Identification of Spen as a crucial factor for Xist function through forward genetic screening in haploid embryonic stem cells," Cell Reports, vol. 12, no. 4, pp. 554-561, 2015.

[36] L. Redrup, M. R. Branco, E. R. Perdeaux et al., "The long noncoding RNA Kcnq1ot1 organises a lineage-specific nuclear domain for epigenetic gene silencing," Development, vol. 136, no. 4, pp. 525-530, 2009.

[37] R. R. Pandey, T. Mondal, F. Mohammad et al., "Kcnq1ot1 antisense noncoding RNA mediates lineage-specific transcriptional silencing through chromatin-level regulation," Molecular Cell, vol. 32, no. 2, pp. 232-246, 2008.

[38] S. J. Hainer and J. A. Martens, "Regulation of chaperone binding and nucleosome dynamics by key residues within the globular domain of histone H3," Epigenetics \& Chromatin, vol. 9, no. 1, p. 17, 2016.

[39] T. Wu, C. Chen, L. Yang et al., "Distinct lncRNA expression profiles in the prefrontal cortex of SD rats after exposure to methylphenidate," Biomedicine \& Pharmacotherapy, vol. 70, pp. 239-247, 2015.

[40] R. Kurokawa, "Promoter-associated long noncoding RNAs repress transcription through a RNA binding protein TLS," Advances in Experimental Medicine and Biology, vol. 722, pp. 196-208, 2011.

[41] I. Cajigas, D. E. Leib, J. Cochrane et al., "Evf2 lncRNA/ BRG1/DLX1 interactions reveal RNA-dependent inhibition of chromatin remodeling," Development, vol. 142, no. 15, pp. 2641-2652, 2015.

[42] E. G. Berghoff, M. F. Clark, S. Chen, I. Cajigas, D. E. Leib, and J. D. Kohtz, "Evf2 (Dlx6as) lncRNA regulates ultraconserved enhancer methylation and the differential transcriptional control of adjacent genes," Development, vol. 140, no. 21, pp. 4407-4416, 2013.

[43] E. Sonkoly, Z. Bata-Csorgo, A. Pivarcsi et al., "Identification and characterization of a novel, psoriasis susceptibility- related noncoding RNA gene, PRINS," Journal of Biological Chemistry, vol. 280, no. 25, pp. 24159-24167, 2005.

[44] X. Sun, M. Li, Y. Sun et al., "The developmental transcriptome sequencing of bovine skeletal muscle reveals a long noncoding RNA, $\operatorname{lncMD}$, promotes muscle differentiation by sponging miR-125b," Biochimica et Biophysica Acta (BBA) - Molecular Cell Research, vol. 1863, no. 11, pp. 28352845, 2016.

[45] C. Zuo, Z. Wang, H. Lu, Z. Dai, X. Liu, and L. Cui, "Expression profiling of lncRNAs in C3H10T1/2 mesenchymal stem cells undergoing early osteoblast differentiation," Molecular Medicine Reports, vol. 8, no. 2, pp. 463-467, 2013.

[46] W. Q. Song, W. Q. Gu, Y. B. Qian, X. Ma, Y. J. Mao, and W. J. $\mathrm{Liu}$, "Identification of long non-coding RNA involved in osteogenic differentiation from mesenchymal stem cells using RNA-Seq data," Genetics and Molecular Research, vol. 14, no. 4, pp. 18268-18279, 2015.

[47] Q. Qu, F. Fang, B. Wu et al., "Potential role of long non-coding RNA in osteogenic differentiation of human periodontal ligament stem cells," Journal of Periodontology, vol. 87, no. 7, pp. e127-e137, 2016.

[48] W. Zhang, R. Dong, S. Diao, J. Du, Z. Fan, and F. Wang, "Differential long noncoding RNA/mRNA expression profiling and functional network analysis during osteogenic differentiation of human bone marrow mesenchymal stem cells," Stem Cell Research \& Theraphy, vol. 8, no. 1, p. 30, 2017.

[49] A. Keniry, D. Oxley, P. Monnier et al., "The H19 lincRNA is a developmental reservoir of miR-675 that suppresses growth and Igf1r," Nature Cell Biology, vol. 14, no. 7, pp. 659-665, 2012.

[50] W. C. Liang, W. M. Fu, Y. B. Wang et al., "H19 activates Wnt signaling and promotes osteoblast differentiation by functioning as a competing endogenous RNA," Scientific Reports, vol. 6, no. 1, article 20121, 2016.

[51] Y. Huang, Y. Zheng, C. Jin, X. Li, L. Jia, and W. Li, "Long noncoding RNA H19 inhibits adipocyte differentiation of bone marrow mesenchymal stem cells through epigenetic modulation of histone deacetylases," Scientific Reports, vol. 6, no. 1, article 28897, 2016.

[52] X. Xiao, T. Zhou, S. Guo et al., "LncRNA MALAT1 sponges miR-204 to promote osteoblast differentiation of human aortic valve interstitial cells through up-regulating Smad4," International Journal of Cardiology, vol. 243, pp. 404-412, 2017.

[53] J. Weng, W. Peng, S. Zhu, and S. Chen, "Long noncoding RNA sponges miR-454 to promote osteogenic differentiation in maxillary sinus membrane stem cells," Implant Dentistry, vol. 26, no. 2, pp. 178-186, 2017.

[54] B. Wei, W. Wei, B. Zhao, X. Guo, and S. Liu, "Long noncoding RNA HOTAIR inhibits miR-17-5p to regulate osteogenic differentiation and proliferation in non-traumatic osteonecrosis of femoral head," PLoS One, vol. 12, no. 2, article e0169097, 2017.

[55] L. Zhu and P. C. Xu, "Downregulated LncRNA-ANCR promotes osteoblast differentiation by targeting EZH2 and regulating Runx2 expression," Biochemical and Biophysical Research Communications, vol. 432, no. 4, pp. 612-617, 2013.

[56] Q. Jia, W. Jiang, and L. Ni, “Down-regulated non-coding RNA (lncRNA-ANCR) promotes osteogenic differentiation of periodontal ligament stem cells," Archives of Oral Biology, vol. 60, no. 2, pp. 234-241, 2015. 
[57] W. Zhuang, X. Ge, S. Yang et al., "Upregulation of lncRNA MEG3 promotes osteogenic differentiation of mesenchymal stem cells from multiple myeloma patients by targeting BMP4 transcription," Stem Cells, vol. 33, no. 6, pp. 19851997, 2015.

[58] Z. Li, C. Jin, S. Chen et al., "Long non-coding RNA MEG3 inhibits adipogenesis and promotes osteogenesis of human adipose-derived mesenchymal stem cells via miR-140-5p," Molecular and Cellular Biochemistry, vol. 433, no. 1-2, pp. 51-60, 2017.

[59] Q. Wang, Y. Li, Y. Zhang et al., "LncRNA MEG3 inhibited osteogenic differentiation of bone marrow mesenchymal stem cells from postmenopausal osteoporosis by targeting miR-133a-3p," Biomedicine \& Pharmacotherapy, vol. 89, pp. 1178-1186, 2017.

[60] F. Wu, C. Zhang, J. Cai et al., "Upregulation of long noncoding RNA HOXA-AS3 promotes tumor progression and predicts poor prognosis in glioma," Oncotarget, vol. 8, pp. 5311053123, 2017.

[61] X. X. Zhu, Y. W. Yan, D. Chen et al., "Long non-coding RNA HoxA-AS3 interacts with EZH2 to regulate lineage commitment of mesenchymal stem cells," Oncotarget, vol. 7, no. 39, pp. 63561-63570, 2016.

[62] C. Jin, Y. Zheng, Y. Huang, Y. Liu, L. Jia, and Y. Zhou, "Long non-coding RNA MIAT knockdown promotes osteogenic differentiation of human adipose-derived stem cells," Cell Biology International, vol. 41, no. 1, pp. 33-41, 2017.

[63] N. Ishii, K. Ozaki, H. Sato et al., "Identification of a novel non-coding RNA, MIAT, that confers risk of myocardial infarction," Journal of Human Genetics, vol. 51, no. 12, pp. 1087-1099, 2006.

[64] Y. Shen, L. F. Dong, R. M. Zhou et al., "Role of long noncoding RNA MIAT in proliferation, apoptosis and migration of lens epithelial cells: a clinical and in vitro study," Journal of Cellular and Molecular Medicine, vol. 20, no. 3, pp. 537548, 2016.

[65] L. Wang, F. Wu, Y. Song et al., "Long noncoding RNA related to periodontitis interacts with miR-182 to upregulate osteogenic differentiation in periodontal mesenchymal stem cells of periodontitis patients," Cell Death \& Disease, vol. 7, no. 8, article e2327, 2016.

[66] C. Jin, L. Jia, Y. Huang et al., "Inhibition of lncRNA MIR31HG promotes osteogenic differentiation of human adipose-derived stem cells," Stem Cells, vol. 34, no. 11, pp. 2707-2720, 2016.

[67] C. F. Liu, W. E. Samsa, G. Zhou, and V. Lefebvre, "Transcriptional control of chondrocyte specification and differentiation," Seminars in Cell \& Developmental Biology, vol. 62, pp. 34-49, 2017.

[68] F. Ou, K. Su, J. Sun et al., “The lncRNA ZBED3-AS1 induces chondrogenesis of human synovial fluid mesenchymal stem cells," Biochemical and Biophysical Research Communications, vol. 487, no. 2, pp. 457-463, 2017.

[69] L. Zhang, S. Chen, N. Bao et al., "Sox4 enhances chondrogenic differentiation and proliferation of human synovium-derived stem cell via activation of long noncoding RNA DANCR," Journal of Molecular Histology, vol. 46, no. 6, pp. 467-473, 2015.

[70] L. Zhang, X. Sun, S. Chen et al., "Long noncoding RNA DANCR regulates $m i R-1305$-Smad 4 axis to promote chondrogenic differentiation of human synovium-derived mesenchymal stem cells," Bioscience Reports, vol. 37, no. 4, article BSR20170347, 2017.
[71] L. Zhang, C. Yang, S. Chen et al., "Long noncoding RNA DANCR is a positive regulator of proliferation and chondrogenic differentiation in human synovium-derived stem cells," DNA and Cell Biology, vol. 36, no. 2, pp. 136-142, 2017.

[72] H. L. Carlson, J. J. Quinn, Y. W. Yang, C. K. Thornburg, H. Y. Chang, and H. S. Stadler, "LncRNA-HIT functions as an epigenetic regulator of chondrogenesis through its recruitment of p100/CBP complexes," PLoS Genetics, vol. 11, no. 12, article e1005680, 2015.

[73] L. Wang, Z. Li, Z. Li, B. Yu, and Y. Wang, "Long noncoding RNAs expression signatures in chondrogenic differentiation of human bone marrow mesenchymal stem cells," Biochemical and Biophysical Research Communications, vol. 456, no. 1, pp. 459-464, 2015.

[74] C. Dou, Z. Cao, B. Yang et al., "Changing expression profiles of lncRNAs, mRNAs, circRNAs and miRNAs during osteoclastogenesis," Scientific Reports, vol. 6, no. 1, article 21499, 2016.

[75] X. Tong, P. C. Gu, S. Z. Xu, and X. J. Lin, "Long non-coding RNA-DANCR in human circulating monocytes: a potential biomarker associated with postmenopausal osteoporosis," Bioscience, Biotechnology, and Biochemistry, vol. 79, no. 5, pp. 732-737, 2015.

[76] J. H. Yoon and M. Gorospe, "Cross-linking immunoprecipitation and qPCR (CLIP-qPCR) analysis to map interactions between long noncoding RNAs and RNA-binding proteins," Methods in Molecular Biology, vol. 1402, pp. 11-17, 2016.

[77] J. H. Yoon, S. De, S. Srikantan et al., "PAR-CLIP analysis uncovers AUF1 impact on target RNA fate and genome integrity," Nature Communications, vol. 5, p. 5248, 2014.

[78] M. B. Friedersdorf and J. D. Keene, "Advancing the functional utility of PAR-CLIP by quantifying background binding to mRNAs and lncRNAs," Genome Biology, vol. 15, no. 1, article R2, 2014.

[79] K. A. Cottrell and S. Djuranovic, "Urb-RIP - an adaptable and efficient approach for immunoprecipitation of RNAs and associated RNAs/proteins," PLoS One, vol. 11, no. 12, article e0167877, 2016.

[80] K. Schaukowitch, J. Y. Joo, and T. K. Kim, "UV-RNA immunoprecipitation (UV-RIP) protocol in neurons," Methods in Molecular Biology, vol. 1468, pp. 33-38, 2017.

[81] J. H. Li, S. Liu, H. Zhou, L. H. Qu, and J. H. Yang, "starBase v2.0: decoding miRNA-ceRNA, miRNA-ncRNA and protein-RNA interaction networks from large-scale CLIP-Seq data," Nucleic Acids Research, vol. 42, no. D1, pp. D92-D97, 2014.

[82] C. Chu and H. Y. Chang, "Understanding RNA-chromatin interactions using chromatin isolation by RNA purification (ChIRP)," Methods in Molecular Biology, vol. 1480, pp. 115123, 2016. 


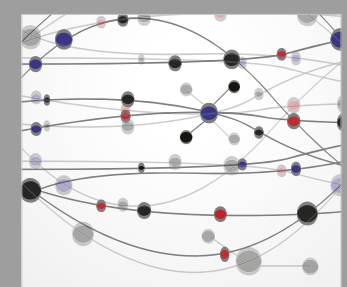

The Scientific World Journal
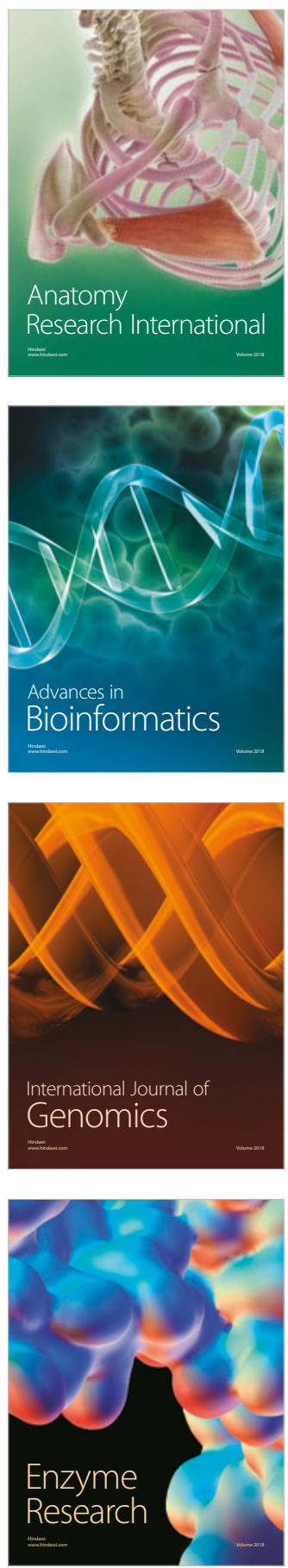
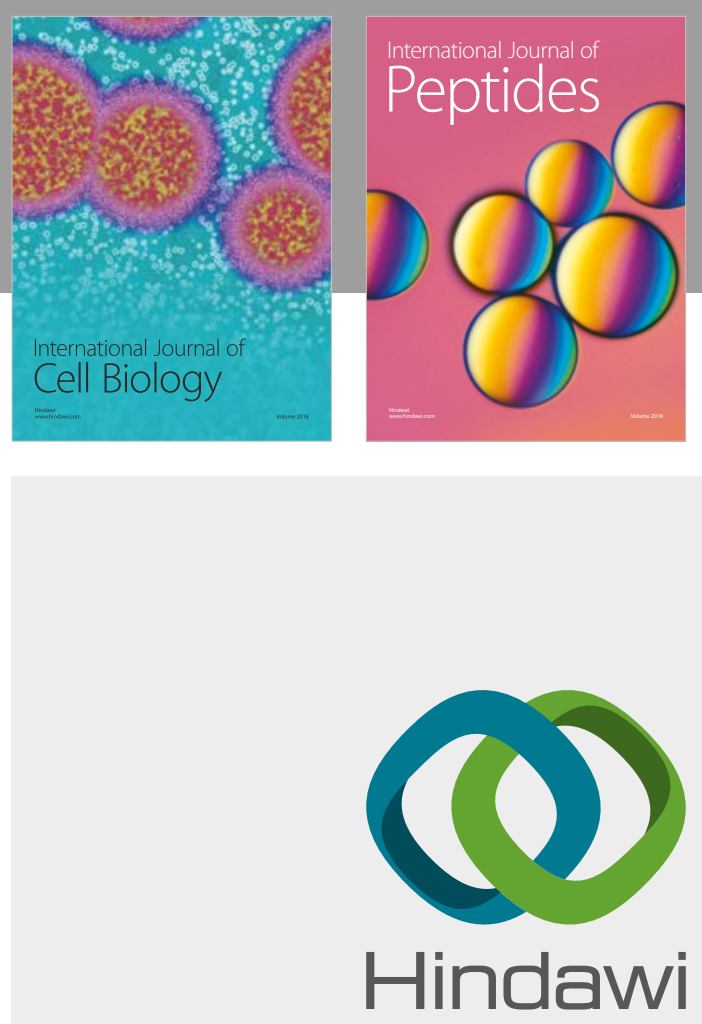

Submit your manuscripts at

www.hindawi.com
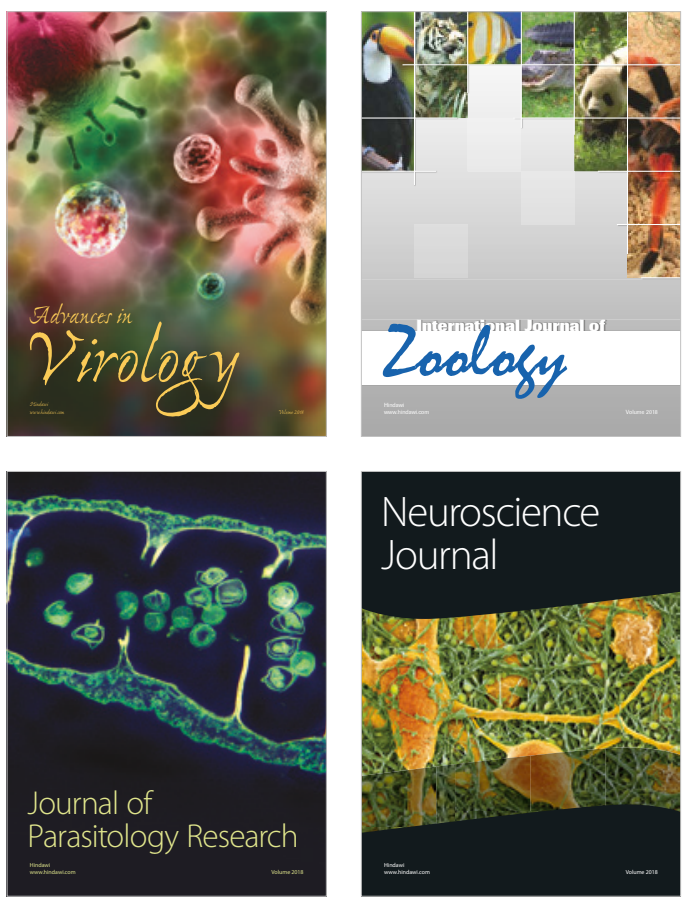
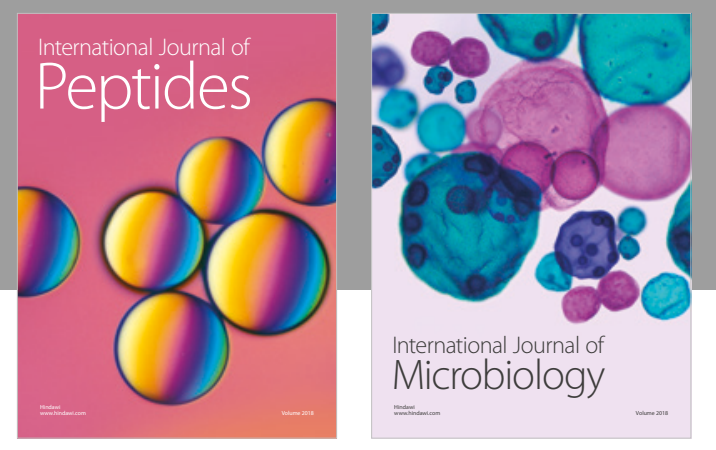

nternational Journal of Microbiology
Journal of
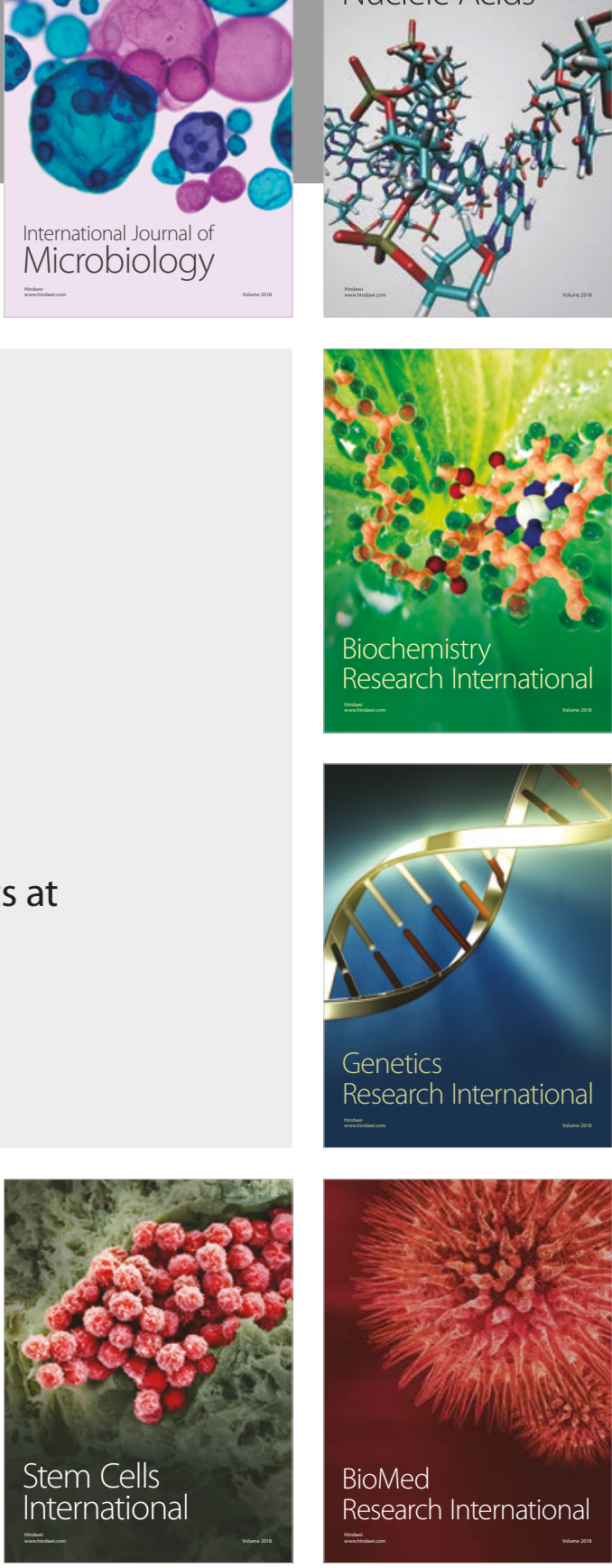
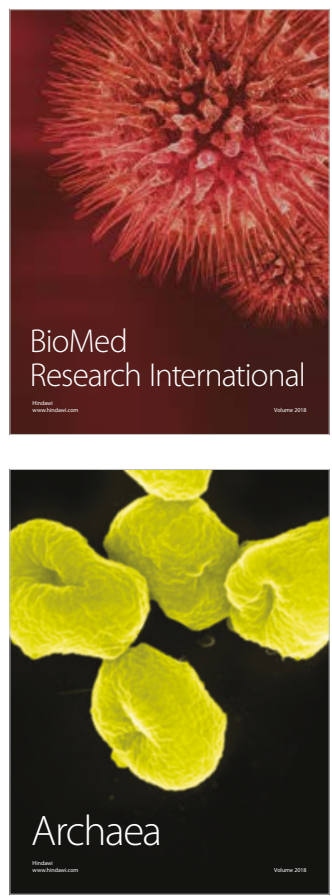